\title{
Stability Boundary for Haptic Rendering: Influence of Human Operator
}

\author{
Thomas Hulin, Carsten Preusche and Gerd Hirzinger
}

\begin{abstract}
Recent analysis on the stability boundary for haptic rendering assumed a stabilizing effect through a human operator holding a haptic device, without considering his/her dynamics directly. This paper derives stability boundaries of a linear model of a haptic system including those dynamics. It shows that all three elements of the human arm modeled as mass-spring-damper system contribute to stability. The haptic system itself is composed of a haptic device colliding with a virtual wall modeled as time-delayed discrete-time spring-damper system. Furthermore, the article proves that the recently found linear stability condition for haptic devices of Gil et al. still holds if a human is holding the haptic device. Finally, a relation to Colgate's passivity condition defining a robustly stable region is given.
\end{abstract}

\section{INTRODUCTION}

An elementary prerequisite for haptic applications is to preserve stability. Numerous theoretical and experimental approaches have been presented in the past that have dealt with ensuring stability for haptic interfaces. The passivity condition of Colgate, et al. in [1] represents one of the most cited theoretical studies towards a common stability condition. Although ensuring passivity of haptic devices is a general approach, it has the disadvantages of being conservative in terms of stability and requiring the presence of mechanical damping.

A more accurate approach was introduced by Hannaford, et al. [2], [3], [4] with their time-domain passivity controller. They introduced a variable damper which eliminates the energy after it was generated by the haptic device.

The exact stability region for haptic walls represented by a virtual spring-damper system was first determined by Salcudean and Vlaar [5]. They considered their haptic device as a simple mass which is actuated by an one sample-step delayed force. For this simplified control loop, they found the stability boundary inside a normalized parameter plane. The human operator was ignored for the stability analysis, as he/she tends to stabilize the system [6], [7].

This approach was enhanced by the authors of [8], considering also time delay as parameter. A more complex model of the haptic system including physical damping was investigated in [9]. It was observed that physical damping is increasing the stable region. This result and the derived stability boundaries are in accordance with previous experiments [10], [11].

This work was partly supported by the VRLab of the Volkswagen AG.

The authors of this article are with the Institute of Robotics and Mechatronics, DLR (German Aerospace Center), D-82234 Wessling, Germany Thomas.Hulinedlr.de

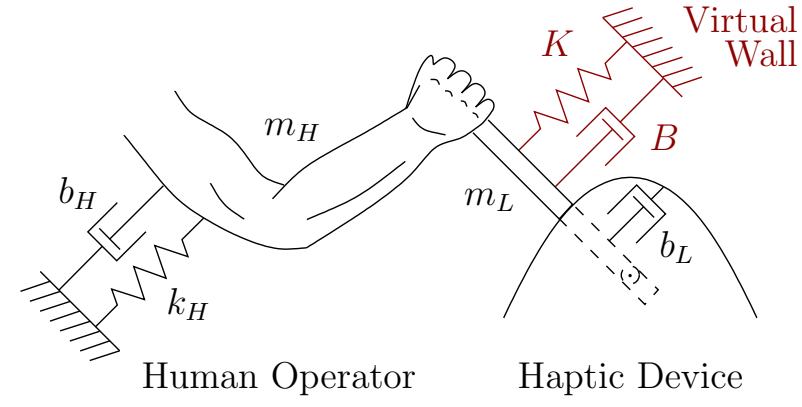

Fig. 1. Simplified model of a human arm interacting with a 1 DoF haptic device.

A similar approach [7] without normalized parameters and time delay resulted in the same stability boundaries. Yet, that article introduced a linear stability condition, which was recently generalized in [12] to admit time delay.

In the stability analyses of the two articles [9] and [12] human dynamics were not taken into account. The present article analyses stability for a haptic system including a massspring-damper model as human arm. It discusses its influence on the stability boundaries of [9] and the linear stability condition of [12].

Section II gives a detailed description of the investigated haptic system. An exact discrete-time equivalent of this system is derived in section III. Section IV investigates the possible range of physical parameters of human arms and haptic devices. On this basis, the exact stability boundaries are presented in section V. Section VI linearizes these boundaries resulting in a linear stability condition. This linear condition is compared to the exact boundaries in section VII, in order to qualify its valid range. Finally, the influence of very high-frequency oscillations on the stability boundaries is discussed in section VIII, finding a relation to Colgate's passivity condition. Finally, section IX summarizes the main results of this article.

\section{SYSTEM DESCRIPTION}

This paper analyses stability for a system that consists of a human operator grabbing an impedance type haptic device. As illustrated in Fig. 1, a single degree of freedom (DoF) mass-spring-damper system is supposed as simplified model for the human arm, with mass $m_{H}$, stiffness $k_{H}$ and viscous damping $b_{H}$. Although it is an approximation, this linear model of the real human has been applied successfully in many theoretical studies [7]. As the following stability analyses describe a situation of the human arm that can be 


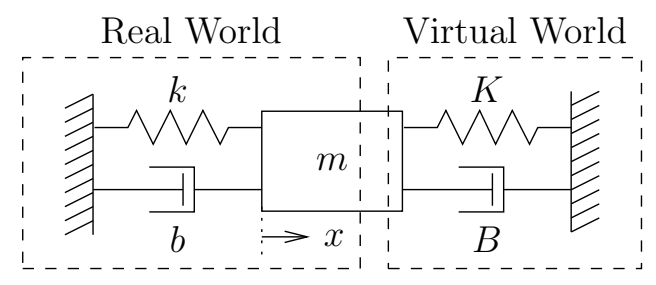

Fig. 2. Physical equivalent of the addressed system.

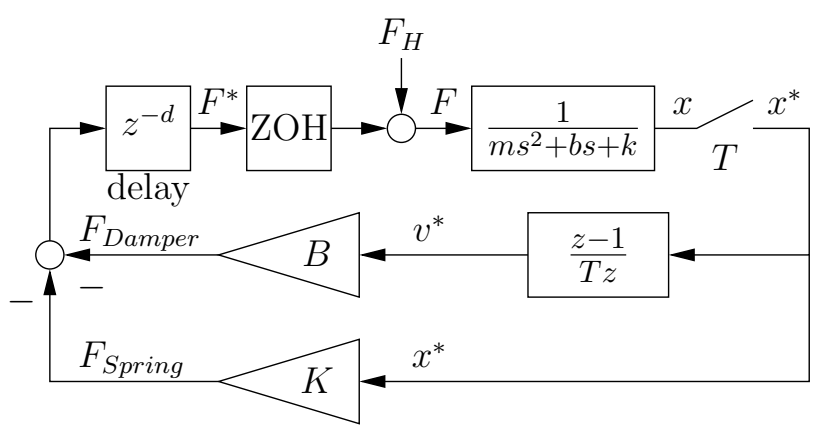

Fig. 3. Control loop of the system.

considered as local in time and space, the linear model is a valid simplification.

The haptic device is a $1 \mathrm{DoF}$ mass $m_{L}$ which is damped by $b_{L}$. A possible compliance of the haptic device is neglected. The human arm's mass $m_{H}$ is directly coupled to the device's inertia $m_{L}$. Therefore, the physical parameters of the haptic device and the human can be combined to

$$
\begin{array}{rrr}
m= & m_{L}+m_{H} \\
b & = & b_{L}+b_{H} \\
k & = & \\
k_{H},
\end{array}
$$

where $m, b$ and $k$ are respectively the effective physical mass, damping and stiffness. Figure 2 shows the physical equivalent of the addressed system.

The haptic device is displaying a virtual wall which is composed of a virtual spring $K$ and damper $B$ (discretetime PD-controller). The force of the virtual wall is a delayed discrete-time signal, with sampling time $T$ and time delay $t_{d}=d T$.

Nonlinear effects like static friction, or quantization and saturation of sensors and actuators are not taken into account. With these assumptions the control loop shown in Fig. 3 can be set up easily. It contains continuous-time (physical stiffness and damping) and discrete-time (virtual wall) elements. Thus, before a stability analysis can be performed, all blocks of the control loop have to be transformed into the same time domain. In analogy to [9], the approach of the present paper transforms the continuous-time blocks into the discrete-time domain.

Remark 1: This paper analyses stability for virtual walls modeled as bilateral spring-damper system. But, these analyses hold also for the case of a human pushing against a (unilateral) virtual wall, such that the position of the mass is not leaving the wall, as this is equivalent to a system
TABLE I

DIMENSIONLESS PARAMETERS

\begin{tabular}{|ccc|}
\hline Parameter & Variable & Dimensionless variable \\
\hline Sampling period & $T$ & - \\
Mass & $m$ & - \\
Virtual stiffness & $K$ & $\alpha=\frac{K T^{2}}{m}$ \\
Virtual damping & $B$ & $\beta=\frac{B T}{m}$ \\
Physical stiffness & $k$ & $\gamma=\frac{k T^{2}}{m}$ \\
Physical damping & $b$ & $\delta=\frac{b T}{m}$ \\
Delay & $t_{d}$ & $d=\frac{t_{d}}{T}$ \\
\hline
\end{tabular}

with a bilateral spring-damper element. If bouncing occurs, i.e. the mass is leaving the virtual wall during an oscillation cycle, the authors expect the found stability condition to be conservative, because the destabilizing discrete-time elements would not affect a whole oscillation cycle. Yet, this case is not investigated in this article.

\section{DISCRETE-TIME EQUIVALENT}

The exact discrete-time equivalent of the continuous-time block in the control loop in Fig. 3, including the zeroorder hold $(\mathrm{ZOH})$ and the sampler, can be determined by some calculations while assuming that the input force $F_{H}$ is constant during one sampling step. The way to determine the exact discrete-time equivalent is shown in detail in [9]. Here, only the result is given as

$$
\begin{gathered}
1 /\left(m s^{2}+b s+k\right) \\
\triangleq \\
\frac{\left(\left(c_{2}+c_{3}-2\right) z+\left(c_{2}+c_{3}-2 e^{-b T / m}\right)\right)\left(b^{2}-4 k m\right)+b c_{1}\left(c_{3}-c_{2}\right)(z-1)}{2 k\left(z^{2}-\left(c_{2}+c_{3}\right) z+e^{-b T / m}\right)\left(4 k m-b^{2}\right)}
\end{gathered}
$$

with

$$
\begin{aligned}
& c_{1}=\sqrt{(b T / m)^{2}-4 k T^{2} / m} \\
& c_{2}=e^{-\left(b T / m+c_{1}\right) / 2} \\
& c_{3}=e^{-\left(b T / m-c_{1}\right) / 2} .
\end{aligned}
$$

The authors of [9] introduced also normalized parameters to simplify their characteristic polynomial, see Table I. For the system investigated in the present article an additional normalized parameter is introduced, the normalized physical stiffness

$$
\gamma=k \cdot T^{2} / m
$$

As it is shown below, introducing this substitution simplifies the resulting characteristic polynomial. With the normalized parameters, (2) becomes

$$
\begin{gathered}
1 /\left(m s^{2}+b s+k\right) \\
\triangleq \\
\frac{T^{2}}{2 m} \frac{\left(\left(2-c_{2}-c_{3}\right) c_{1}+\left(c_{2}-c_{3}\right) \delta\right) z+\left(2 e^{-\delta}-c_{3}-c_{2}\right) c_{1}+\left(c_{3}-c_{2}\right) \delta}{c_{1} \gamma\left(z^{2}-\left(c_{2}+c_{3}\right) z+e^{-\delta}\right)}
\end{gathered}
$$

with

$$
\begin{aligned}
& c_{1}=\sqrt{\delta^{2}-4 \gamma} \\
& c_{2}=e^{-\left(\delta+c_{1}\right) / 2} \\
& c_{3}=e^{-\left(\delta-c_{1}\right) / 2}
\end{aligned}
$$


This exact discrete-time equivalent allows to derive easily the closed-loop transfer function from force $F^{*}$ to position $x^{*}$ (compare Fig. 3) as

$$
G_{x}(z)=\frac{T^{2} z^{1+d}}{m} \cdot \frac{n(z)}{p(z)}
$$

with

$$
\begin{aligned}
n(z) & =\left(\left(c_{2}+c_{3}-2\right) c_{1}-\left(c_{2}-c_{3}\right) \delta\right) z \\
& +\left(c_{2}+c_{3}-2 e^{-\delta}\right) c_{1}+\left(c_{2}-c_{3}\right) \delta
\end{aligned}
$$

and

$$
\begin{aligned}
p(z) & = \\
& \left(\left(c_{3}+c_{2}-2\right) c_{1}+\left(c_{3}-c_{2}\right) \delta\right)(\alpha+\beta) z^{2} \\
& +\left(\left(\left(c_{3}+c_{2}-2 e^{-\delta}\right) c_{1}+\left(c_{2}-c_{3}\right) \delta\right) \alpha\right. \\
& \left.\quad+2\left(\left(1-e^{-\delta}\right) c_{1}+\left(c_{2}-c_{3}\right) \delta\right) \beta\right) z \\
& -2\left(z^{2}-z\left(c_{3}+c_{2}\right)+e^{-\delta}\right) c_{1} \gamma z^{1+d} \\
& +\left(\left(2 e^{-\delta}-c_{3}-c_{2}\right) c_{1}+\left(c_{3}-c_{2}\right) \delta\right) \beta .
\end{aligned}
$$

The normalized characteristic polynomial $p(z)$ depends only on the five dimensionless parameters $\alpha, \beta, \gamma, \delta$ and $d$. The mass $m$ and the sampling time $T$ dropped out due to the applied normalization rules of Table I.

Remark 2: If $k=0$, the discrete-time transfer function (2) can be simplified to

$$
H_{x}(z)=\frac{x^{*}}{F^{*}}=\frac{T}{b(z-1)}-\frac{m\left(1-e^{-T b / m}\right)}{b^{2}\left(z-e^{-T b / m}\right)} .
$$

This corresponds to the transfer function determined in [9].

\section{PARAMETER RANGE}

This section investigates the ranges for the two normalized parameters $\gamma$ and $\delta$ for existing haptic systems. Gil et al. [7] listed several values for mass, stiffness and damping of a human arm that were used in literature. For all of them the relations of stiffness to mass and damping to mass is located inside the ranges

$$
\begin{aligned}
& 0 \leq k_{H} / m_{H}<710 \mathrm{~s}^{-2} \\
& 0 \leq b_{H} / m_{H}<12.6 \mathrm{~s}^{-1},
\end{aligned}
$$

if the case of an ungrabbed device $k_{H}=b_{H}=0$ is also permitted (s means seconds). To determine the ranges of the normalized physical parameters $\gamma$ and $\delta$, recall their definitions first:

$$
\begin{aligned}
& \gamma=T^{2} \cdot k_{H} /\left(m_{L}+m_{H}\right) \\
& \delta=T \cdot\left(b_{L}+b_{H}\right) /\left(m_{L}+m_{H}\right) .
\end{aligned}
$$

It can easily be checked that the two inequalities

$$
\begin{aligned}
& \gamma \leq T^{2} \cdot k_{H} / m_{H} \\
& \delta \leq T \cdot\left(b_{L} / m_{L}+b_{H} / m_{H}\right)
\end{aligned}
$$

hold for positive masses and dampers. Combining (11) with (13), and taking into account that $b_{L} / m_{L}$ is usually smaller than 0.625 for existing haptic devices (see [13]), yields

$$
\begin{aligned}
& 0 \leq \gamma<T^{2} \cdot 710 \mathrm{~s}^{-2} \\
& 0 \leq \delta<T \cdot 13.225 \mathrm{~s}^{-1}
\end{aligned}
$$

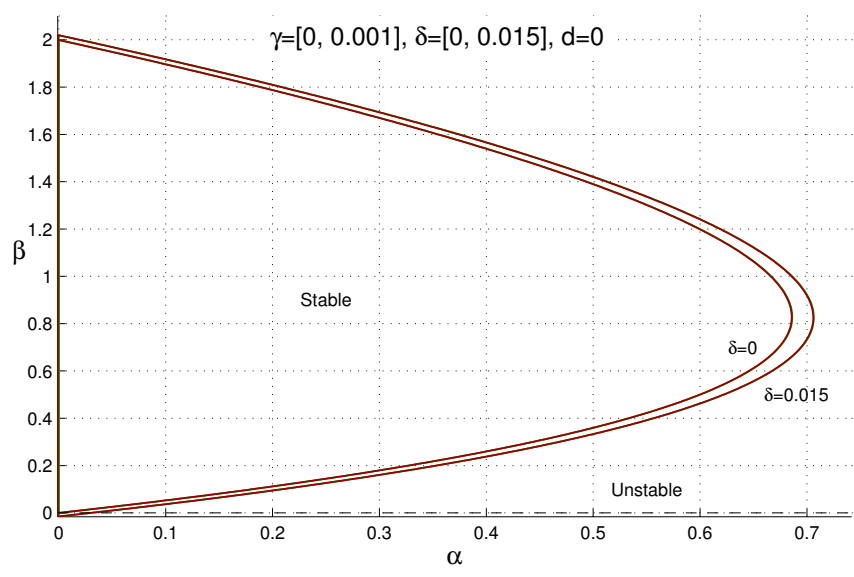

Fig. 4. Exact stability boundary in the $(\alpha, \beta)$-plane for $d=0$ and for the limits of the parameter range $\gamma=[0,0.001]$ and $\delta=[0,0.015]$. The effect of $\gamma$ on the stability boundary is not visible in this scale.

To account for the fact that reliable values for the physical parameters of human operators and haptic devices are in general quite imprecise values, the parameter range will be extended. For example, the mass of both a human arm and a serially-linked haptic device can vary remarkably depending on their actual configurations.

Finally, it will be assumed that the sampling time for haptic systems is limited from above at $T \leq 1 \mathrm{~ms}$, because this is a widely accepted limit. Therefore, the following parameter range will be considered:

$$
\begin{aligned}
& 0 \leq \gamma<1 \cdot 10^{-3} \\
& 0 \leq \delta<15 \cdot 10^{-3} .
\end{aligned}
$$

The following section determines the stability boundary for this parameter range.

\section{STABILITY BOUNDARIES}

A stability check of the investigated system can easily be performed by computing the roots of the discrete-time transfer function (7). The system is stable if all roots of $p(z)$ are located inside the unit circle. The stable region is obtained if two parameters of $p(z)$ are gridded, and all the others are fixed.

For the limits of the possible ranges of the two physical parameters $\gamma$ and $\delta$, Fig. 4 shows the stability boundaries inside the $(\alpha, \beta)$-plane for a fixed delay $d=0$. The effect of the physical damping $\delta$ is the same than it was observed in [9] - physical damping increases the stable region. Yet, it seems that the physical stiffness $\gamma$ does not affect the stability boundary at all. But, a close-up around the origin illustrates that an increasing $\gamma$ shifts the left vertical boundary of the stable region to $\alpha=-\gamma$, see Fig. 5. The lower boundary in Fig. 5 is not visibly influenced by $\gamma$.

For larger delay the stable region becomes smaller [9]. Therefore, the influence of $\gamma$ inside its bounds becomes visible in the overall shape of the stable region for larger delays, see Fig. 6 for $d=1$ and $d=2$. 

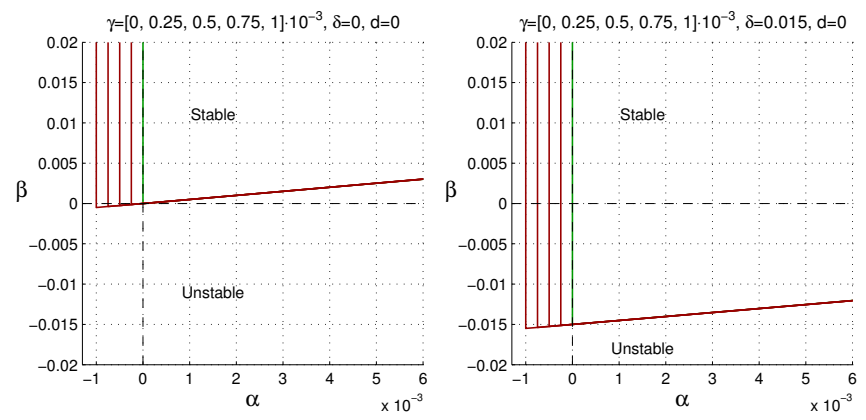

Fig. 5. Close-up of Fig. 4 around the origin. Physical stiffness $\gamma$ is shifting the left boundary to $\alpha=-\gamma$ and not visibly influencing the bottom boundary.
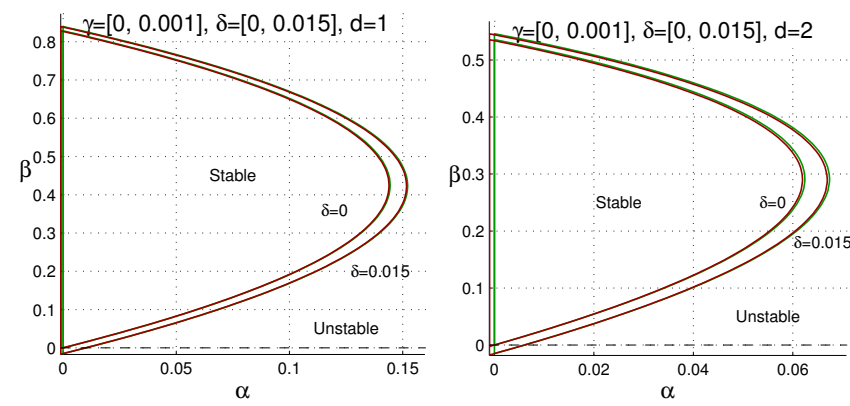

Fig. 6. Stability boundary in the $(\alpha, \beta)$-plane for $d=1$ (left) and $d=$ 2 (right) for the limits of the parameter range $\gamma=[0,0.001]$ and $\delta=$ $[0,0.015]$.

To conclude, physical stiffness and damping originated from a human are increasing the stable region. Due to the normalization rules, also the mass $m_{H}$ is contributing to stability. Therefore, if modeled as linear mass-spring-damper system, all three elements (the mass, the spring and the damper) of a human operator are stabilizing an impedance type haptic system.

\section{LINEAR STABILITY CONDITION}

This section derives a linear stability condition for the investigated system. In accordance with [12], the linear condition will be obtained by applying the Routh-Hurwitz criterion.

If the effect of sampling and hold is approximated by a delay of half a sampling step $T / 2$, for the characteristic equation of the control loop in Fig. 3 yields

$$
1+\frac{K+B s}{m s^{2}+b s+k} e^{-\left(t_{d}+T / 2\right) s}=0 .
$$

Further, substituting the effective delay $t_{r}=t_{d}+T / 2$ and approximating $e^{-t_{r} s} \approx \frac{1}{1+t_{r} s}$ yields

$$
m t_{r} s^{3}+\left(b t_{r}+m\right) s^{2}+\left(k t_{r}+b+B\right) s+k+K=0 .
$$

By applying the Routh-Hurwitz criterion and assuming that the physical parameters $m, T, t_{d}, b, k$ are positive, three inequalities result,

$$
\begin{gathered}
b+B>-k t_{r} \\
K>-k
\end{gathered}
$$

$$
b t_{r}\left(b+B+k t_{r}\right)+m\left(b+B-K t_{r}\right)>0 .
$$

The last inequality can be rewritten as

$$
b+B+\underbrace{b t_{r}\left(b+B+k t_{r}\right) / m}_{\epsilon}>K t_{r} .
$$

Due to previous assumption $T \leq 1 \mathrm{~ms}$, for small delay $d$, and under condition (18), the summand $\epsilon$ can be neglected and inequality (21) simplifies to

$$
K<\frac{b+B}{t_{r}},
$$

which is exactly the linear stability condition found in [12]. The virtual stiffness $K$ must be smaller than the sum of damping $b+B$ devided by the total delay $t_{d}+T / 2$. Note that physical stiffness $k$ does not influence equation (22). It shifts only the lower limit of the virtual stiffness to $K>-k$. Furthermore, equation (18) is implied in (19) and (22).

The two stability conditions (19) and (22) hold well for haptic systems for which previous approximations and assumptions are valid, i.e. the effective delay $t_{r}$ must be small compared to the oscillation time of the mass $m$ and small compared to the fraction $m / b$.

\section{VALIDITY OF LINEAR STABILITY CONDITION}

Due to an exact conversion into the discrete-time domain it was possible to determine the stability boundaries of the system shown in Fig. 2. This section compares the linear stability condition (22) to the exact boundaries. The linear condition in normalized parameters reads

$$
\beta+\delta>\alpha(1 / 2+d)
$$

Similar to [12], the relative error of the virtual stiffness $\alpha$ between both boundaries is considered. This error is defined as

$$
\left|\left(\alpha_{l i n}-\alpha\right) /(\alpha+\gamma)\right|
$$

for $\alpha$ and $\alpha_{\text {lin }}$ located on the exact, respectively linear stability boundary for the same $\beta$. Fig. 7 shows the exact (solid) and the linear condition (dashed) in the same plots. It visualizes also the points at which the relative error reaches a level of $2 \%$ (circles) and $5 \%$ (squares).

The linear condition that was suggested in [12] for haptic systems with physical damping, still holds if physical stiffness of the human arm is introduced. Furthermore, the valid range of the linear condition is nearly not affected by physical stiffness (compare left with right plots in Fig. 7): The values for $\alpha$ at which the limits of $2 \%$ and $5 \%$ are reached differ less than $0.2 \%$ between $\gamma=0$ and $\gamma=0.001$ for the values used in Fig. 7. For larger delay, the relative influence is stronger, as the stable region becomes smaller.

Although the valid range of the linear condition ends at numerically small values on the $\alpha$-axis, the non-normalized values for the virtual stiffness at which the linear condition holds are huge. This is due to the square of the sampling rate in the normalization rule of $\alpha$ and the typically fast sampling frequency ( $T \leq 1 \mathrm{~ms}$ ) in haptic systems. 

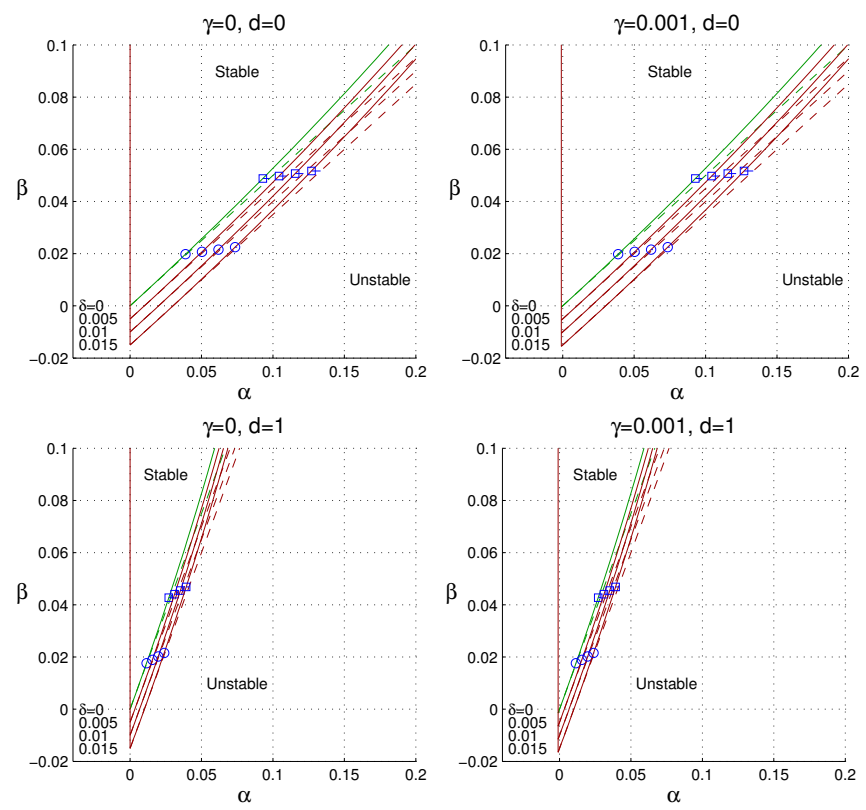

Fig. 7. Validity of the linear stability condition. Exact stability boundaries (solid) for places where the relative error of the linear condition (23) (dashed) is equal to $2 \%$ (circles) and equal to $5 \%$ (squares), for $\delta \in$ $[0,0.005,0.01,0.015]$ and $\gamma=0$ (left) resp. $\gamma=0.001$ (right). At the top two plots holds $d=0$, at the bottom $d=1$.

\section{NYQUIST FREQUENCY}

Previous sections assumed small values for physical stiffness, $\gamma \leq 0.001$, resulting in eigenfrequencies that are far from Nyquist frequency. Although a human operator cannot apply such high stiffness, it is still interesting to observe the behavior of the stability boundaries for large $\gamma$, because a connection to Colgate's passivity condition [1] can be found.

If the mass' oscillation exceeds Nyquist frequency $\omega_{N}=$ $\pi / T$, those oscillations cannot be observed correctly in the discrete-time domain. Therefore, this section investigates the stability of the haptic system for frequencies up to the Nyquist frequency. is

The oscillation frequency of a mass-spring-damper system

$$
\omega=\sqrt{\frac{k}{m}-\frac{b^{2}}{4 m}},
$$

for $k>b^{2} / 4$. Substituting the physical by the normalized parameters yields

$$
\omega=\frac{\sqrt{\gamma-\delta^{2} / 4}}{T} .
$$

Thus, the system is oscillating with Nyquist frequency if

$$
\gamma-\delta^{2} / 4=\pi^{2}
$$

\section{A. No Time-Delay $d=0$}

Fig. 8 shows the stability boundaries for $\delta=0, d=0$ and $\gamma$ from 0 to $\frac{1}{4} \pi^{2}$ (left) respectively $\pi^{2}$ (right), to reach half or full Nyquist frequency. When increasing physical stiffness up to half Nyquist frequency $\gamma=\frac{1}{4} \pi^{2}$, the stable region moves completely to the left-half pane $(\alpha \leq 0)$ for half Nyquist frequency.
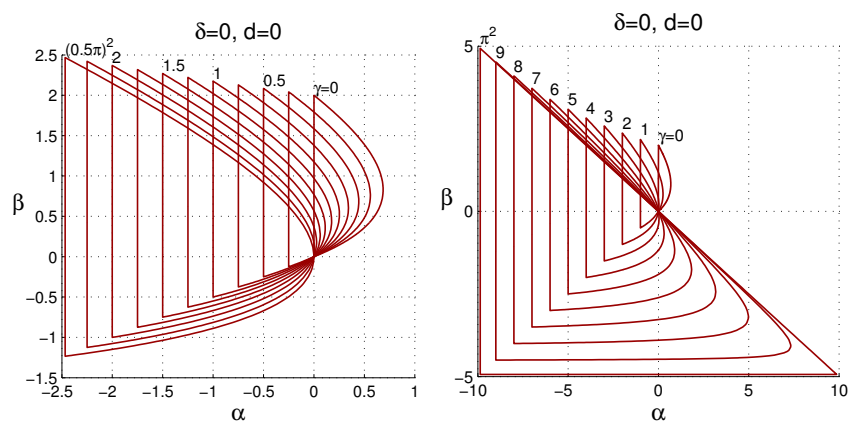

Fig. 8. The stability boundaries for $\delta=0, d=0$ and for parameter values of $\gamma$ such that the system is reaching half (left plot; $0 \leq \gamma \leq(0.5 \pi)^{2}$ ) or full (right plot; $0 \leq \gamma \leq \pi^{2}$ ) Nyquist frequency.
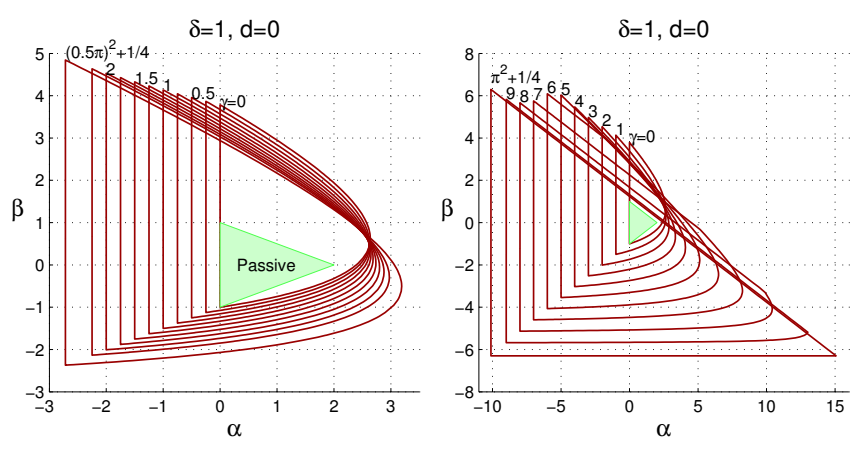

Fig. 9. Same case than in Fig. 8, but for $\delta=1$. Colgate's passivity condition is specifying the passive region, plotted as green triangle.

A further increase of $\gamma$ results in a triangular stable region exactly at the Nyquist frequency for $\gamma=\pi^{2}$. It is interesting that a maximum virtual stiffness $\alpha$ requires for negative virtual damping $\beta$. A robustly stable region is the intersection of the stable regions for all $\gamma$. For the values in Fig. 8, the robustly stable region is the point of origin.

The stable region increases for larger physical damping, as shown exemplarily in Fig. 9 for $\delta=1$. Again, at Nyquist frequency, i.e. for $\gamma=\pi^{2}+1 / 4$, the shape of the stable region is a triangle. The robustly stable point in the $(\alpha, \beta)$ plane has increased to a robustly stable region. Apparently, this robust region must contain or be equal to the passive region.

The passive region is given by Colgate's passivity condition in [1] for $d=0$, which can be written with the normalized parameters:

$$
\delta>\frac{\alpha}{2}+|\beta|,
$$

for $\alpha \geq 0, T>0$ and $m>0$. The passive region is also shown in Fig. 9. Although both are quite similar, the passive region is located completely inside the robustly stable region.

Remark 3: Colgate used different dimensionless parameters in [1] (e.g. $\tau \triangleq 1 / \gamma$ ). His results for - what he calls - uncoupled stability correspond to the stable regions for $\gamma=0, \delta=0$ and $d=0$ of the present article. Yet, the results for his spring stability differ from those of the present article. 

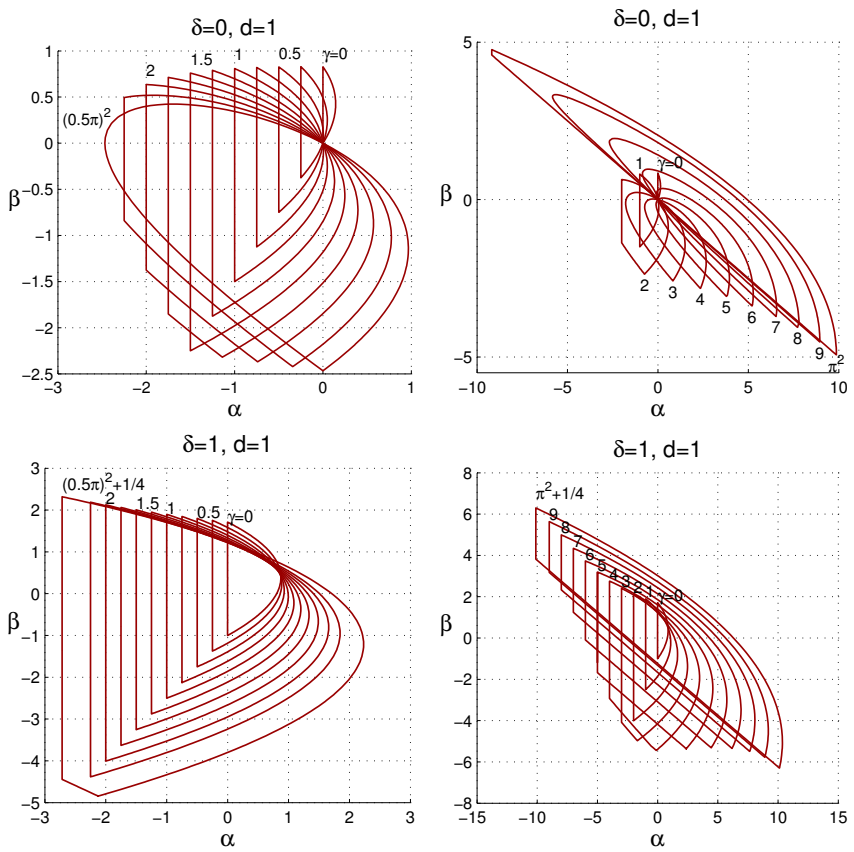

Fig. 10. Same case as in Fig. 8, but for $d=1$. For the two plots on the top holds $\delta=0$, and for the two at the bottom $\delta=1$.

\section{B. Time-Delay of one Sampling Step $d=1$}

For lager delays, the shape of the stable region differs from previous case. Fig. 10 exemplarily shows the progression of the stable region for $d=1$ and increasing values of $\gamma$ up to the Nyquist frequency. Again, for systems with no physical damping $(\delta=0)$ exists only a robustly stable point in the origin.

From the two plots at the top of Fig. $10(\delta=0, d=1)$, it can be seen that the stable region moves to the left-half plane $(\alpha \leq 0)$ for smaller values of $\gamma$ compared to previous case of $d=0$. For $\gamma=(\pi / 4)^{2}$ it is lying completely inside the left-half plane.

Independent of the time-delay $d$ (also for $d=0$ ), the whole stable region is limit stable for $\gamma=\pi^{2}$ and $\delta=0$, as the system has a pole at $z=-1$.

Remark 4: To reach Nyquist frequency for an exemplary haptic device similar to the PHANToM device $(m=0.1 \mathrm{~kg}$, $T=0.001 \mathrm{~s}, b=1.5 \mathrm{Ns} / \mathrm{m}, d=0$ ), a physical stiffness of $k=\pi^{2} m / T^{2}+b^{2} / 4 m \approx 987.0 \mathrm{kN} / \mathrm{m}$ would be necessary. This value can be reached for collisions of the haptic device with the environment, but by far not through a human arm.

\section{CONCLUSIONS}

The present article performed a stability analysis of a haptic system interacting with a human arm that was modeled as linear mass-spring-damper system. The haptic device was colliding against a virtual wall that consisted of a timedelayed discrete-time spring-damper system.

The paper derived a linear stability condition, which was compared to exact stability boundaries. Inside the parameter range of real haptic devices, this linear condition holds well. The stiffness of the human arm has no effect on the linear condition. Yet, as stated before all three elements of the arm contribute to stability and increase the stable region. As they are usually very small, the effect of human stiffness and damping is negligible compared to the influence of the human arm's mass.

Finally, the influence of a very large physical stiffness on the exact stability boundaries was discussed, and Colgate's passive region was found to be located inside a robustly stable region. A minimum amount of physical damping is needed to obtain a region which is robustly stable against any physical stiffness, especially during collision between the haptic device and a stiff real environment.

For future work, open issues would be worthwhile to investigate. The most important topic would be to include nonlinear effects like unilateral virtual walls (instead of its bilateral spring-damper representation), static friction and quantization effects, to obtain more precise results. Furthermore, more complex dynamics could be included in the analysis, like a more realistic model of a human operator or a model of the haptic device including motor dynamics.

\section{ACKNOWLEDGMENTS}

The authors would like to thank Dr. Naim Bajcinca for his valuable ideas and the fruitful discussions.

\section{REFERENCES}

[1] J. E. Colgate and G. Schenkel, "Passivity of a class of sampled-data systems: Application to haptic interfaces," Journal of Robotic Systems, vol. 14 , pp. 37-47, 1997.

[2] B. Hannaford and J. Ryu, "Time-domain passivity control of haptic interfaces," in Proc. of the IEEE Int. Conf. on Robotics and Automation (ICRA), 2001, pp. 1863-1869.

[3] J. Ryu, Y. Kim, and B. Hannaford, "Sampled and continuous time passivity and stability of virtual environments," in Proc. of the IEEE Int. Conf. on Robotics and Automation (ICRA), 2003, pp. 822-827.

[4] J.-H. Ryu, C. Preusche, B. Hannaford, and G. Hirzinger, "Time domain passivity control with reference energy behavior," IEEE Transactions on Control Systems Technology, 2005.

[5] S. Salcudean and T. Vlaar, "On the emulation of stiff walls and static friction with a magnetically levitated input/output device," ASME Journal of Dynamic Systems, Measurement and Control, March 1997.

[6] R. J. Adams and B. Hannaford, "Stable haptic interaction with virtual environments," IEEE Trans. on Robotics and Automation, vol. 15, no. 3, pp. 465-474, June 1999.

[7] J. Gil, A. Avello, A. Rubio, and J. Flórez, "Stability analysis of a 1 dof haptic interface using the routh-hurwitz criterion," IEEE Transactions on Control Systems Technology, pp. 583-588, 2004.

[8] T. Hulin, C. Preusche, and G. Hirzinger, "Stability boundary and design criteria for haptic rendering of virtual walls," in Proc. of the 8th International IFAC Symposium on Robot Control, Bologna, Italy, September 2006

[9] — "Stability boundary for haptic rendering: Influence of physical damping," in Proc. IEEE/RSJ Int. Conf. on Intelligent Robots and Systems (IROS), Beijing, China, October 2006, pp. 1570-1575.

[10] J. Colgate and J. Brown, "Factors affecting the z-width of a haptic display," in Proc. of the IEEE Int. Conf. on Robotics and Automation, May 1994, pp. 3205-3210.

[11] J. Mehling, J. Colgate, and M. Peshkin, "Increasing the impedance range of a haptic display by adding electrical damping," in Proc. of the IEEE WorldHaptics Conference (WHC), Pisa, Italy, March 2005, pp. 257-262.

[12] J. Gil, E. Sánchez, T. Hulin, C. Preusche, and G. Hirzinger, "Stability boundary for haptic rendering: Influence of damping and delay," in Proc. of the IEEE Int. Conf. on Robotics and Automation (ICRA), Rome, Italy, April 2007.

[13] N. Diolaiti, G. Niemeyer, F. Barbagli, and J. Salisbury, "Stability of haptic rendering: Discretization, quantization, time delay, and coulomb effects," IEEE Transactions on Robotics, vol. 22, no. 2, pp. 256-268, April 2006. 